\title{
Asymptomatic "Exposers" and "Infectors" are Sources of Infection by SARS-CoV-2: Analysis based on Family Clustering
}

Yu He

Wuhan Union Hospital

\section{Yuanyuan Guo}

Liyuan Hospital

Ping Liu

Liyuan Hospital

Rui Li

Wuhan Union Hospital https://orcid.org/0000-0002-9407-8591

Liu Ouyang

Wuhan Union Hospital

Siyuan Wang

Liyuan Hospital

Dehao Fu ( $\nabla$ fudehao@hust.edu.cn )

Union Hospital

Research article

Keywords: COVID-19, Asymptomatic infectors, Asymptomatic exposers, family clustering infection

Posted Date: August 13th, 2020

DOI: https://doi.org/10.21203/rs.3.rs-42516/v1

License: (c) (i) This work is licensed under a Creative Commons Attribution 4.0 International License.

Read Full License 


\section{Abstract}

Background: Previous studies have documented the clinical characteristics of patients with Coronavirus disease 2019(COVID-19) and presented evidence of person-to-person transmission. Limited data are available for patients with asymptomatic infections. Some asymptomatic carriers, whom we characterize as "exposers" or "infectors", may be responsible for family clustering of COVID-19.

Methods: A questionnaire survey and follow-up survey based on media reports were used to assess familial clustering of SARS-CoV-2 infection induced by asymptomatic exposers/infectors. Individual data were collected for all members of each tracked family. A transmission map was then drawn for each family.

Results: Our study of 5 families indicated that individuals with no obvious symptoms of COVID-19, regardless of the PCR results, transmitted the virus to other family members who were community contained at home and had no contact with other infected individuals. There was one death case in Family No.3.

Conclusion: Asymptomatic exposers/infectors of SARS-CoV-2 were all middle-aged (average age: $44.4 \pm$ 14.9 years) who had no symptoms but had the ability to disseminate the virus. Medical staff participating in treatment of COVID-19 cases all had a high risk of infection, they should be quarantined so as to protect their families. The morbidity and mortality of Case 3.2 remind us that although these asymptomatic infected people have no symptoms, they are also infectious. It is not ruled out that the subsequent infected people are seriously ill or even die. Therefore, we should not take it lightly.

\section{Background}

Since the first report of an outbreak of pneumonia with an unknown cause in Wuhan, Hubei Province (China) $)^{1-3}$, there has been considerable controversy on the origin of the causative virus (SARS-CoV-2, also referred to as HCoV-19) ${ }^{4}$, the epidemiology, and the pathogenesis of this novel coronavirus disease (COVID-19). The COVID-19 outbreak is now characterized as a pandemic because the virus has spread worldwide. Globally, as of 10:00 AM CEST, 6 May 2020, there have been 3,588,773 confirmed cases of COVID-19, including 247,503 deaths, reported to WHO. ${ }^{5}$

Why was the outbreak so swift and violent? There was a report of an asymptomatic confirmed German patient who transmitted the virus to other people on January 30 , suggesting that asymptomatic carriers were also infectious. ${ }^{4}$ Subsequently, an article in the February 2 issue of Science questioned this notion, and concluded that the evidence was still insufficient. ${ }^{5}$ Thus, there is controversy regarding the spread of this virus by asymptomatic carriers. As front-line workers in Wuhan, we found some phenomena among our colleagues that could shed light on this notion. In particular, there was evidence of an index case without any signs or symptoms, despite exposed to SARS-CoV-2, whose family members developed 
symptoms of COVID-19 following contact with the index case. A follow-up of these cases by chest computed tomography (CT) and virus PCR test confirmed infection by SARS-CoV-2.

We classified, for the first time, individuals who had exposed to the confirmed COVID-19 cases, with no symptoms and negative CT and PCR results as "asymptomatic exposers", and those who had no symptoms and negative CT but laboratory-confirmed SARS-CoV-2 infection as "asymptomatic infectors". There were few case reports of virus transmission by asymptomatic carriers by the news media in China. We tracked these cases to investigate the possible role of asymptomatic "exposers" or "infectors" as sources of infection, which may help to prevent the transmission of the COVID-19 epidemic.

\section{Methods}

\section{Data collection}

A questionnaire with a two-dimensional code was designed that could be efficiently distributed through the mobile network. This questionnaire rapidly spread among WeChat groups and social media, was completed in the WeChat applet. This also allowed collection of background data and identified targeted households by extensive screening. The study subjects also provided informed consent through the media.

\section{Inclusion and exclusion criteria}

A family was included if: (a) two or more family members tested positive by PCR; (b) the asymptomatic index case had no signs or symptoms of infection but had close contact with a confirmed patient (highintensity exposure) in the epidemic area, even without any protective measures; (c) family members tested positive, but the index case tested negative (asymptomatic "exposer") or positive (asymptomatic "infector").

A family was excluded if: (a) every member of the familial cluster had signs or symptoms of COVID-19 (i.e., all of them were symptomatic); (b) the asymptomatic family member was not the index case responsible for the familial cluster outbreak.

\section{Protection measures for study subjects and researchers}

Appropriate protection measures were adopted for all study subjects. The study was deemed safe for all vulnerable subjects (minors, pregnant women, students, persons with no or limited capacities) and would not cause additional harm to these individuals.

Because the subjects contacted by the researchers could not be excluded as asymptomatic "exposers" or "infectors", all researchers adopted level-2 personal protective equipment(PPE) during events that required contact with the subjects (collecting specimens, etc.).

\section{Data analysis and Statistics}


After analysis of case data with the above inclusion and exclusion criteria, 5 groups of eligible familial clusters were included. Initially, these families answered a detailed questionnaire about the specific route and the course of infection and the changes of symptoms. The focus of this study was to track asymptomatic "exposers" or "infectors". Nucleic acid PCR tests and chest CT scans were taken for all the family members. Some subjects who were tested previously were exempted. The confidentiality of all personal data, including medical files, personal and family information, was properly protected.

We collected the diagnostic data of all patients and compare the diagnostic efficiency of CT and nucleic acid tests. Data were collected retrospectively and reported as means \pm SDs. $P$ value below 0.05 was considered significant. All data were analyzed using SPSS 20.0 software.

\section{Ethical statement}

This study was approved by the Ethics Committee of Union Hospital Affiliated to Tongji Medical College of Huazhong University of Science and Technology.

\section{Results}

\section{Epidemiologic characteristics}

Two hundred and forty questionnaires were collected and one family was selected for inclusion. Among the patients in the fever clinic, the symptomatic infected cases were tracked and included in the questionnaire for screening and one family met the criteria for inclusion. Through the news media, three families clustering outbreak due to asymptomatic "exposers" or "infectors" were included and back tracking. All five families were included in the questionnaire for detailed investigation. We analyzed data of the five familial clusters, each of which had evidence of confirmed infection. (Fig1A,1C,1E, Fig2A 2C)

\section{Analysis of transmission and the epidemic process}

According to our definition, there were 7 asymptomatic exposers or infectors in the 5 families (Tab.1). Most of them were middle-aged (average age: $44.4 \pm 14.9$ years). The asymptomatic index cases in two families were medical workers who were exposed to subsequently confirmed cases, and did not take necessary protective measures during the very early stage of the epidemic. Thus, these individuals had high-intensity exposure. Although the onset of symptoms in asymptomatic exposers/infectors were generally relatively mild, but there was one death in one of the families.

In family No.1(Fig.1B), Case 1.1 is classified as the asymptomatic exposer defined by us, the PCR test was always negative despite repeated many times. However, from the analysis of the epidemic transmission process, Case 1.1 was highly suspected, although without any sign or symptoms, and transmitted to the family. Of course, we did not rule out the influence of false negative nucleic acid test on the results. There was even one case with 8 times of negative nucleic acid test before the confirmation of COVID-19 reported by in China. As to Case 1.1 there was no symptom all the time, no more nucleic acid test was carried out during the follow-up survey. As to Case1.3 and Case1.4, they have typical symptoms 
and CT imaging of lung, but the nucleic acid test has always been negative, so it can only be classified as suspected infectors, or clinical confirmed cases (Fifth edition of diagnosis and Treatment Guide) ${ }^{6}$.

In family No.3 (Fig.1F), Case 3.2 and Case 3.3 were both old people at home, without related contact history. Case 3.1 has been to the epidemic area and had contact with some confirmed patients, but he has no symptoms. Case 3.1 was found in the screening of close contacts after the onset of Case 3.3, and only one positive test was found in five times, which fully shows the concealment and variability of the virus after infection. However, Case 3.2 died shortly after the onset of the disease.

In family No.5 (Fig.2D.), Case5.2 is a $65 \mathrm{y} / 0$ woman who is the aged well living at home. She went to hospital for cough and fever $\left(38.7^{\circ} \mathrm{C}\right)$ in January 30th. Chest CT examination showed classic groundglass opacities. According to clinical manifestations, she was highly suspected to be infected, and sooner confirmed by the nucleic acid test in January 31 st. After the elderly was admitted, her neighbors, their loved ones and others were diagnosed in succession (the nucleic acid test was positive for asymptomatic infection), and the elderly had no history related to epidemic area, nor had they ever contacted with the confirmed or suspected patients. After investigation, her son-in-law Case 5.1 had a contact history with people from the epidemic area on January 17 to 19 , and attend family reunion at his mother-in-law's home on January 21. Although Case 5.1 has not been infected, the process of epidemic transmission is confirmed by the positive virus nucleic acid test. Other families had similar transmission and the epidemic process (Fig1D and Fig2B).

\section{Comparison of efficiency of lung CT and virus nucleic acid PCR detection}

The average time from onset of symptoms to hospital admission with positive chest CT results was 1.21 \pm 0.92 days, and the average time from onset of symptoms to positive PCR results was $2.77 \pm 2.76$ days $(P<0.05) . \otimes$ Fig $3 \rrbracket$

\section{Discussion}

\section{Viral virulence, infectivity and asymptomatic infection}

Available data indicated that SARS-CoV-2 is less virulent than other coronaviruses, such as SARS-CoV and MERS-CoV, and that symptoms are milder at the initial onset of disease, but it's much more contagious. ${ }^{6-8}$ Zou et al. ${ }^{9}$ used PCR to measure the SARS-CoV-2 viral load in upper respiratory tract samples and their results showed symptomatic and asymptomatic patients had similar viral loads. Hoehl et $\mathrm{al}^{10}$ reported that in an effort to evacuate 126 people from Wuhan to Frankfurt, a symptom-based screening process was ineffective in detecting COVID-19 in 2 patients who confirmed for the COVID-19 infection later by throat swabs. So that special attention and efforts are necessary for the rapid detection of SARS-CoV-2 infection and the isolation of COVID-19 cases.

\section{Discovery of asymptomatic carriers}


Asymptomatic patients have the risk of transmission which is difficult to prevent and control. It is difficult to be found in the population and patients with mild or atypical symptoms do not think they are infected and refuse to visit doctors. The existing asymptomatic carriers are mainly found by active screening of close contacts, investigation of infection source, investigation of clustering epidemic situation and active detection of personnel in high-risk areas. A familial cluster of 5 patients with COVID-19 in Anyang, Henan Province, China, had contact with an asymptomatic family member back from the epicenter-Wuhan (Fig1C and Fig1D). ${ }^{11}$

\section{Prevalence of asymptomatic SARS-CoV-2 infection}

Because asymptomatic carriers have the potential to spread infections, it is important to know the prevalence of asymptomatic infections. Mizumoto $\mathrm{K}$ et $\mathrm{al}^{12}$ conducted statistical models to derive the delay-adjusted asymptomatic proportion of infections on the Diamond Princess cruise ship hosting 3,711 people and their estimated asymptomatic proportion was $17.9 \%$ (95\% confidence interval (Cl): 15.5$20.2 \%)$. Considering the high proportion of the elderly, who is more vulnerable to the coronavirus, on the ship, he suspects that the rate in the general population may be closer to a recently derived estimate of $30.8 \%$ (95\% confidence interval (Cl): 7.7\%-53.8\%) from data of Japanese citizens evacuated from Wuhan. ${ }^{13}$

An epidemiology group in China published a retrospective analysis of 889 cases with asymptomatic infections and found that they accounted for $1.2 \%$ of the total. ${ }^{14}$ The data of China do not include asymptomatic infections as confirmed cases before April $1,2020^{15}$. Wang et al ${ }^{16}$ developed a susceptible-exposed-infectious-recovered model to assess the extent of invisible infection. Individual-level data on 25,961 laboratory confirmed COVID-19 cases reported through February 18, 2020 were extracted from the municipal Notifiable Disease Report System of Wuhan City and found that at least $59 \%$ of infected cases were unascertained in Wuhan, potentially including asymptomatic and mild-symptomatic cases. In addition, the hidden virus carriers have not yet been detected cannot be excluded.

\section{Clinical characteristics of asymptomatic carriers}

Asymptomatic exposers/infectors have no signs or symptoms, and may even have no positive chest CT results. Hu et al ${ }^{17}$ investigated the clinical characteristics of 24 cases with asymptomatic infection screened from close contacts of COVID-19 patients (or suspected patients) in Nanjing, none of the 24 asymptomatic cases presented any obvious symptoms while nucleic acid screening. 5 cases $(20.8 \%)$ developed symptoms (fever, cough, fatigue, etc.) during hospitalization. 20 (50.0\%) cases showed typical CT images of ground-glass chest and $5(20.8 \%)$ presented stripe shadowing in the lungs. The remaining 7 $(29.2 \%)$ cases showed normal CT image and had no symptoms during hospitalization. These 7 cases were younger (median age: 14.0 years; $P=0.012$ ) than the rest.

\section{Causes of viral dissemination by asymptomatic carriers}


A German research group published their research in medRxiv, on March 8th, ${ }^{18}$ they found that SARS-CoV2 could actively replicate in the upper respiratory tract. In other words, when the symptoms were mild, the virus was released easily by coughing or sneezing. This process was called "virus abscission" and spread to others. Of course, the chance of transmission caused by pathogen discharge in vitro is relatively less than that of confirmed cases due to the absence of clinical symptoms such as cough and sneeze.

After close contact with confirmed cases or tourism history of epidemic area, some patients with symptoms enter the diagnostic process, most of them do not have symptoms. Some of which were diagnosed as asymptomatic infectors by positive Nucleic acid PCR result, while others with negative result should be classified as asymptomatic exposers. In view of this situation, we propose a treatment process for asymptomatic infectors and asymptomatic exposers. (Fig4)

How can an individual carrying negative PCR results cause infections in close contacts? There are three possibilities. Firstly, the results may be "false negative". Secondly, the person may have transmitted the virus before developing immunity, so the viral load at the time of PCR testing was below the detection limit. Thirdly, viable viruses may have been on their skin or clothing and spread among close contacts. It is suggested that their self-segregation will play an important role in the epidemic prevention and control (Fig.4) .

\section{Family clusters of infection caused by medical staff}

Healthcare workers and elderly people had higher attack rates and severity risk increased with age and special efforts are needed to protect vulnerable populations, including healthcare workers, elderly and children ${ }^{16}$. In our study, two families were exposed to index cases who worked in a hospital, which was not an isolated phenomena that appeared by accident. During the very earlier stages of the epidemic when the cause was unknown, some medical care providers did not have necessary protective measures. Therefore, the staff participating in the care of COVID-19 patients in the hospital should be considered as a high-risk group, and they should be quarantined so as to protect their families (elderly and children) even if they have no obvious symptoms.

\section{Interpretation and response to false negative results}

Xie X et al ${ }^{19}$ reported some patients with positive chest CT findings may present with negative results of RT-PCR. How did this happen? Firstly, in principle, the specificity is high while the sensitivity is relatively low, so there is the possibility of false negative results. Secondly, the PCR kit has a detection limit and the result is positive only when the number of viruses in the sample exceeds that limit. Thirdly, apart from sampling technique, samples from the lower respiratory tract are best while most samples were from the upper respiratory tract considering inconvenient and invasive. Fourthly, other factors such as preservation, transportation of the sample and so on. The presence of some false negative results is inevitable. However, it is necessary to improve our understanding and correctly interpret the results. So that the PCR detection can play the greatest role in diagnostic virology, rather than misleading to the opposite direction. 


\section{Value of CT imaging}

Generally, CT provided more rapid detection of COVID-19 than PCR $\otimes$ Fig.3®, during the early stage of the epidemic, there were few false negative PCR results because of a shortage of PCR kits. It was reported that positive cases could appear even after 2 or 3 negative PCR results ${ }^{20-21}$. Particularly, in the fifth edition of the NHC guidelines for diagnosis and treatment ${ }^{6}$, in high incidence areas, CT alone was regarded as the standard for clinical diagnosis, and these cases were classified into clinical diagnosis cases, such as Case1.3 and Case1.4.(Fig.1) Moreover, the correlation between PCR positivity and symptoms of COVID-19 is not significant. A CT examination can provide early identification of pulmonary parenchymal lesions, which is helpful for guiding treatment decisions. Lung improvements (based on CT) can allow caregivers to adjust the treatment process and even assess prognosis. The results from CT imaging are more important than those from PCR testing for determining the seriousness of an infection $20-21$. However, PCR detection is necessary to establish etiology and confirmation of the diagnosis because CT imaging cannot distinguish among different causes of pneumonia.

\section{Conclusion}

We can draw some conclusions from this study. Firstly, asymptomatic exposers or infectors, are mostly middle-aged people with strong immunity system, but are nonetheless likely to disseminate the virus. Secondly, asymptomatic "exposers" or "infectors" in two of the analyzed families were medical workers, indicating that health care workers participating in the treatment of COVID-19 cases are under high risk. Even if these individuals have no any symptoms, they should be quarantined from their families to prevent family outbreaks. Thirdly, it is necessary to strictly quarantine and observe all people from an epidemic area, rather than simply screening body temperature. We recommend routine performance of PCR testing and chest CT scans. Fourthly, the onset of symptoms caused by asymptomatic "exposers" or "infectors" were generally relatively mild, but there was one death in one of the families. This require further study, but the harm of asymptomatic infection should not be ignored. Finally, urgent measures are needed to contain mild and asymptomatic cases that are exacerbating the pandemic, strong social distancing is the only way to stop the spread of the virus, such as closure of schools, the cancellation of public gatherings, and the keeping of people at home and away from public places.

In general, the focus of this study is on the family cluster outbreaks caused by asymptomatic "exposers" or "infectors", and only 5 families are traced, which is the inadequacy of this study. On March 30, 2020, the official website of the National Health Commission of China began to release the number of asymptomatic infections. As of April 14, 6764 cases of asymptomatic cases have been reported in China, including 588 imported cases from abroad. There were 1297 confirmed cases, 251 of which were imported from abroad, 4444 were removed from medical observation, 109 of which were imported from abroad. 1023 asymptomatic cases still under medical observation, 228 of which were imported from abroad. ${ }^{22}$ Compared with 82601 confirmed cases in China, the proportion of asymptomatic cases is $7.6 \%$, which is different from $18-59 \%$ estimated in other literatures. ${ }^{12,13,16}$ 


\section{Abbreviations}

\begin{tabular}{|ll|}
\hline COVID-19 & The novel coronavirus disease \\
\hline SARS-CoV-2 & Severe acute respiratory syndrome coronavirus 2 \\
\hline CT & Computed tomography \\
\hline PCR & Polymerase Chain Reaction \\
\hline WHO & World Health Organization \\
\hline PPE & personal protective equipment \\
\hline MERS-CoV & Middle East Respiratory Syndrome coronavirus \\
\hline Cl & Confidence Interval \\
\hline
\end{tabular}

\section{Declarations}

\section{Ethics approval and consent to participate}

This study was approved by the Ethics Committee of Union Hospital Affiliated to Tongji Medical College of Huazhong University of Science and Technology. And verbal consent was obtained from the patients. As is known to all,the COVID-19 has swept the whole China. Many cities have been locked down for a long time. So it's very difficult for us to make effective contact with all the people involved. Then we submitted an application to the ethics committee for exemption from signing an informed consent and the application has been approved.

\section{Consent for publication}

Multiple patients involved were informally asked if they felt the results reported herein were reflective of their illness experience to check the validity of the findings.

\section{Availability of data and materials}

All data generated or analysed during this study are included in this published article.

\section{Competing interests}

All authors have completed the ICMJE uniform disclosure form at www.icmje.org/coi_disclosure.pdf and declare: no financial relationships with any organisations that might have an interest in the submitted work in the previous three years; no other relationships or activities that could appear to have influenced the submitted work

\section{Funding}


We have no fund.

\section{Authors' contributions}

Dehao Fu conceived and designed the manuscript with Ye He and Yuanyuan Guo. Ping Liu, Siyuan Wang, Liu Ouyang and Rui Li in charge of data collection. Yu He and Dehao Fu wrote the manuscript. Yuanyuan Guo polished the language.

\section{Acknowledgements}

The authors will thank Prof Youxiu Wei for his great help in data collection.

\section{Reference}

1. WHO. Novel coronavirus - China. Jan 12, 2020. http://www.who. int/csr/don/12-january-2020-novelcoronavirus-china/en/ (Accessed Jan 19, 2020)

2. National Health Commission's briefing on the pneumonia epidemic situation. Released on 23 Feb 2020 (in Chinese).

(http://www.nhc.gov.cn/yjb/s7860/202001/9614b05a8cac4ffabac10c4502fe517c.s html)

3. Li Q, Guan X, Wu P, et al. Early transmission dynamics in Wuhan, China, of novel coronavirus-infected pneumonia. N Engl J Med. 2020. (Published online Jan 29. 2020. DOI: 10.1056/NEJMoa2001316)

4. Camilla R, Mirjam S, Peter S. Transmission of 2019-nCoV Infection from an Asymptomatic Contact in Germany. N Engl J Med. 2020. (Published online Jan 30, 2020 DOI: 10.1056/ NEJMc2001468)

5. Kai K. Study claiming new coronavirus can be transmitted by people without symptoms was flawed. Science. 2020. (Pubulished on line Feb.2 and Retrieved Feb. 4, 2020, from https://www.sciencemag.org/news/2020/02/paper-non-symptomatic-patient-transmittingcoronavirus-wrong)

6. NHC囚The diagnosis and treatment of novel coronavirus-caused pneumonia (trial edition 5 in Chinese). http://www.gov.cn/zhengce/zhengceku/202002/05/5474791/files/de44557832ad4be1929091dcbcfca891.pdf

7. Wang W, Tang J, Wei F. Updated understanding of the outbreak of 2019 novel coronavirus (2019nCoV) in Wuhan, China. J Med Virol. 2020. (Published online ahead of print, 2020 Jan 29 DOI:10.1002/jmv.25689)

8. Park SE. Epidemiology, virology, and clinical features of severe acute respiratory syndrome coronavirus-2 (SARS-CoV-2; Coronavirus Disease-19) [published online ahead of print, $2020 \mathrm{Apr}$ 2]. Clin Exp Pediatr. 2020;10.3345/cep.2020.00493. doi:10.3345/cep.2020.00493

9. Zou L, Ruan F, Huang M, et al. SARS-CoV-2 Viral Load in Upper Respiratory Specimens of Infected Patients. N Engl J Med. 2020;41(2):NEJMc2001737. 10.1056/NEJMc2001737

10. HoehI S, Berger A, Kortenbusch M et al. Evidence of SARS-CoV-2 Infection in Returning Travelers from Wuhan, China. N Engl J Med.2020 Feb 18. doi: 10.1056/NEJMc2001899. [Epub ahead of print] 
11. Bai Y, Yao L, Wei T, et al. Presumed Asymptomatic Carrier Transmission of COVID-19 [published online ahead of print, 2020 Feb 21]. JAMA. 2020;10.1001/jama.2020.2565.

doi:10.1001/jama.2020.2565

12. Mizumoto K, Kagaya K, Zarebski A, et al . Estimating the asymptomatic proportion of coronavirus disease 2019 (COVID-19) cases on board the Diamond Princess cruise ship, Yokohama, Japan, 2020. Euro Surveill. 2020;25(10):2000180. doi:10.2807/1560-7917.ES.2020.25.10.2000180

13. Nishiura $H$, Kobayashi $T$, Suzuki A, et al. Estimation of the asymptomatic ratio of novel coronavirus infections (COVID-19) [published online ahead of print, 2020 Mar 13]. Int J Infect Dis. 2020;S12019712(20)30139-9. doi:10.1016/j.ijid.2020.03.020

14. The Novel Coronavirus Pneumonia Emergency Response Epidemiology Team. An update on the epidemiological characteristics of novel coronavirus pneumonia (COVID-19). Chinese Journal of Epidemiology. DOI: 10.3760/cma.j.issn.0254-6450.2020.02.002.

15. David C. Scientists question China's decision not to report symptom-free coronavirus cases. Nature NEWS .20 FEBRUARY 2020

16. Wang C, Liu L, Hao X, et al. Evolving Epidemiology and Impact of Non-pharmaceutical Interventions on the Outbreak of Coronavirus Disease 2019 in Wuhan, China. Preprint at medRxiv

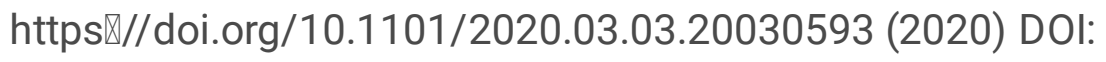
https://doi.org/10.1101/2020.03.03.20030593

17. Hu Z, Song C, Xu C, et al. Clinical characteristics of 24 asymptomatic infections with COVID-19 screened among close contacts in Nanjing, China [published online ahead of print, $2020 \mathrm{Mar} 4$ ]. Sci China Life Sci. 2020;10.1007/s11427-020-1661-4. doi:10.1007/s11427-020-1661-4

18. Roman W, Victor MC, Wolfgang G, et al. Clinical presentation and virological assessment of hospitalized cases of coronavirus disease 2019 in a travel-associated transmission cluster. Preprint at medRxiv 2020.03.05.20030502; DOI: https://doi.org/10.1101/2020.03.05.20030502

19. Xie X, Zhong Z, Zhao W, Zheng C, Wang F, Liu J. Chest CT for Typical 2019-nCoV Pneumonia: Relationship to Negative RT-PCR Testing [published online ahead of print, 2020 Feb 12]. Radiology. 2020;200343. doi:10.1148/radiol.2020200343

20. Wang D, Hu B, Hu C, et al. Clinical Characteristics of 138 Hospitalized Patients With 2019 Novel Coronavirus-Infected Pneumonia in Wuhan, China. JAMA. 2020 Feb 7. doi: 10.1001/jama.2020.1585

21. Pan, Y, Guan, H. Imaging changes in patients with 2019-nCov. Eur Radiol (2020). https://doi.org/10.1007/s00330-020-06713-z

22. Disease prevention and Control Bureau of the National Health Commission of the people's Republic of China. Questions about novel coronavirus asymptomatic infection prevention and control http://www.nhc.gov.cn/jkj/s3578/202003/718c79c96f3e46409dd49303d41a00ef.shtml

\section{Tables}

Tab. 1. Characteristics of the five families. 


\begin{tabular}{|c|c|c|c|c|c|}
\hline & $\begin{array}{l}\text { Family } \\
1\end{array}$ & $\begin{array}{l}\text { Family } \\
2\end{array}$ & $\begin{array}{l}\text { Family } \\
3\end{array}$ & $\begin{array}{l}\text { Family } \\
4\end{array}$ & $\begin{array}{l}\text { Family } \\
5\end{array}$ \\
\hline Total individuals, $\mathrm{n}$ & 5 & 9 & 8 & 6 & 3 \\
\hline $\begin{array}{l}\text { Age range, years } \\
(<40 / 40 \text { to } 65 />65)\end{array}$ & $2 / 2 / 1$ & $1 / 5 / 3$ & $1 / 6 / 1$ & $1 / 5 / 0$ & $1 / 0 / 2$ \\
\hline Gender (male/female) & $1 / 4$ & $3 / 6$ & $3 / 5$ & $1 / 5$ & $2 / 1$ \\
\hline Basic diseases (yes/no) & $1 / 4$ & $2 / 7$ & $1 / 7$ & $0 / 6$ & $1 / 2$ \\
\hline $\begin{array}{l}\text { Light or none / normal / heavy (critical) / } \\
\text { death }\end{array}$ & $2 / 2 / 1 / 0$ & $2 / 6 / 1 / 0$ & $4 / 3 / 1 / 0$ & $1 / 3 / 2 / 0$ & $1 / 0 / 1 / 1$ \\
\hline
\end{tabular}

\section{Figures}



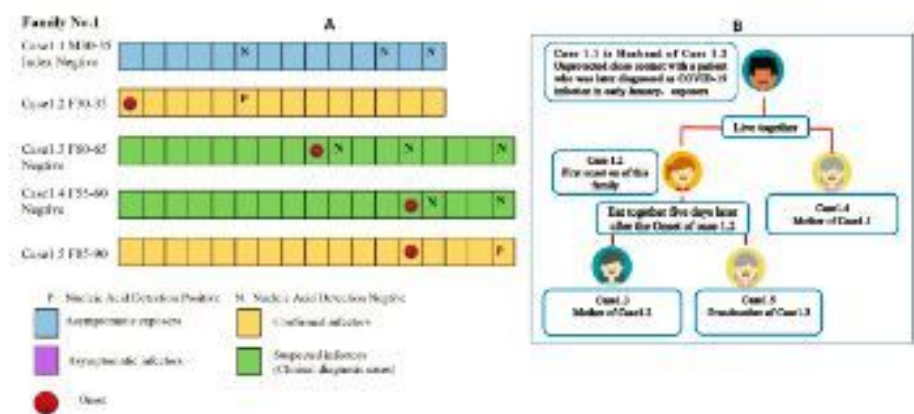

-
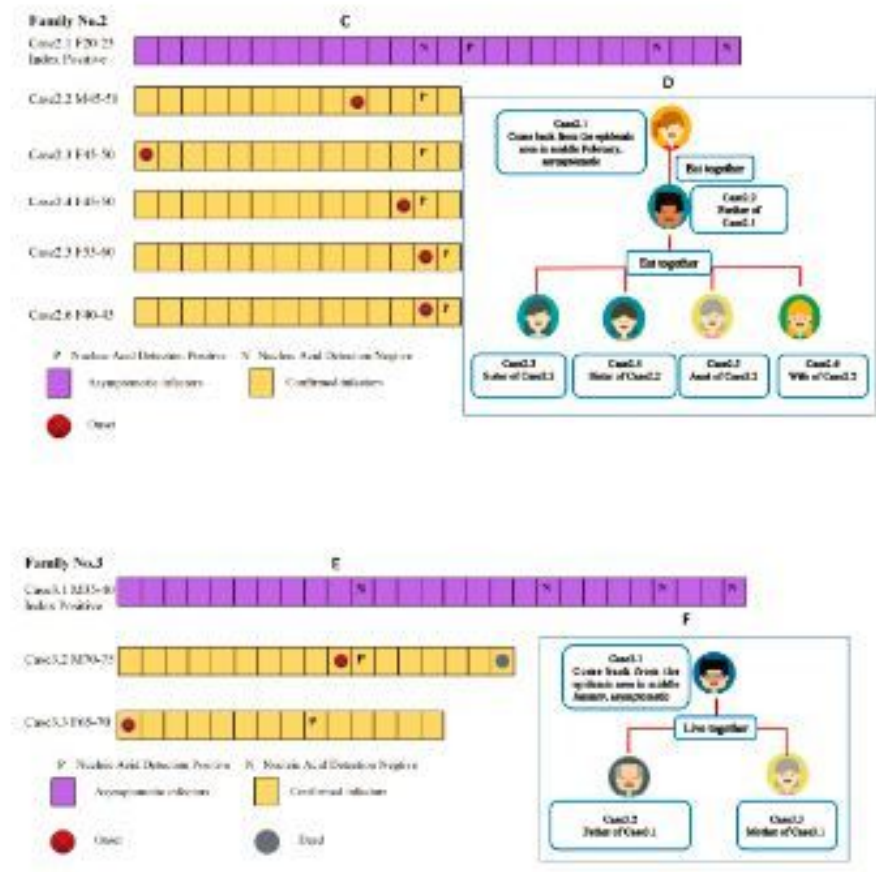

\section{Figure 1}

A: Cases with SARS-CoV-2 infection in family/cluster No.1; B: Transmission map of family/cluster No.1; C: Cases with SARS-CoV-2 infection in family/cluster No.2; D: Transmission map of family/cluster No.2; E: Cases with SARS-CoV-2 infection in family/cluster No.3; F: Transmission map of family/cluster No.3; 

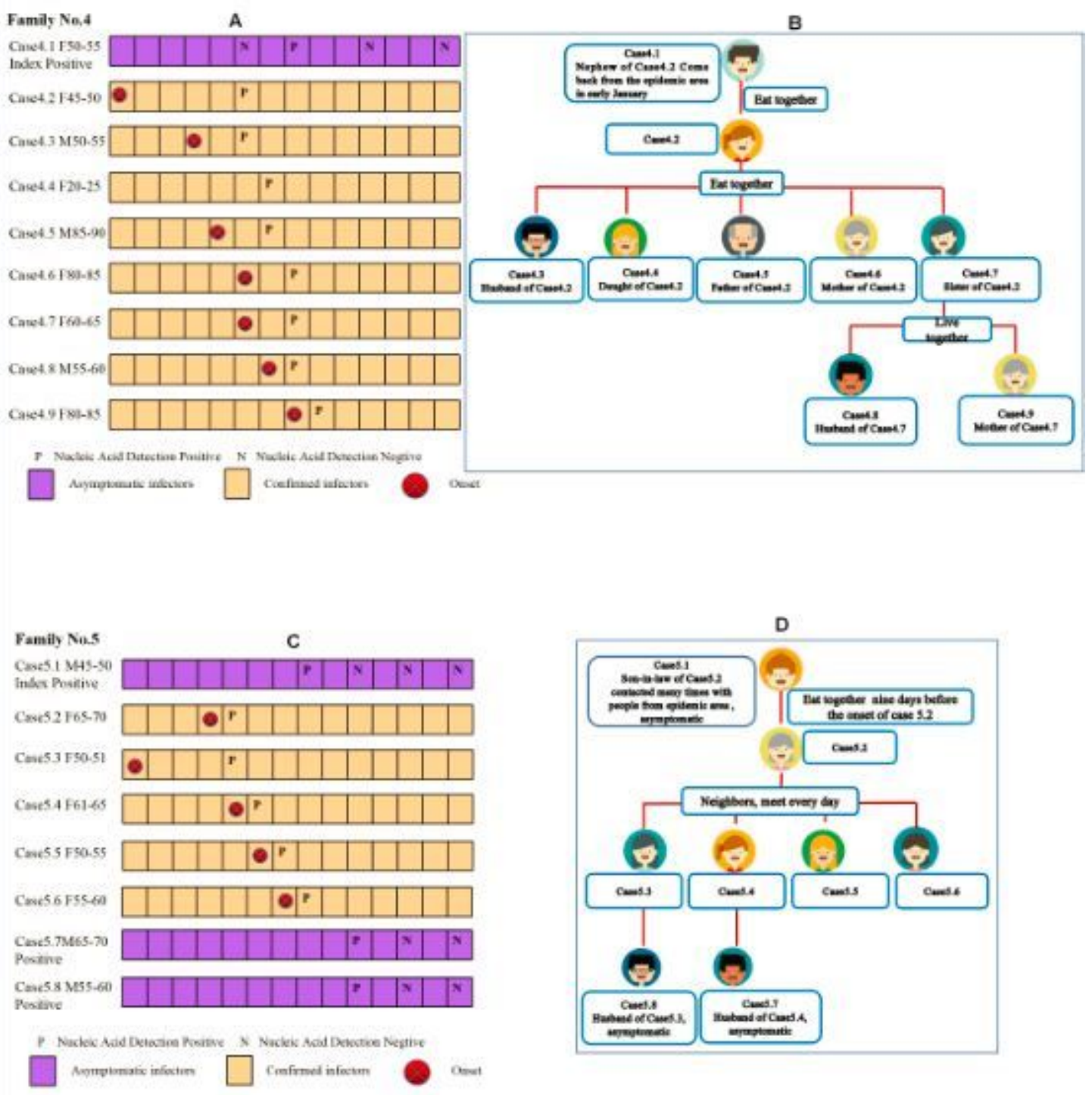

Figure 2

A: Cases with SARS-CoV-2 infection in family/cluster No.4; B: Transmission map of family/cluster No.4; C: Cases with SARS-CoV-2 infection in family/cluster No.5; D: Transmission map of family/cluster No.5. 


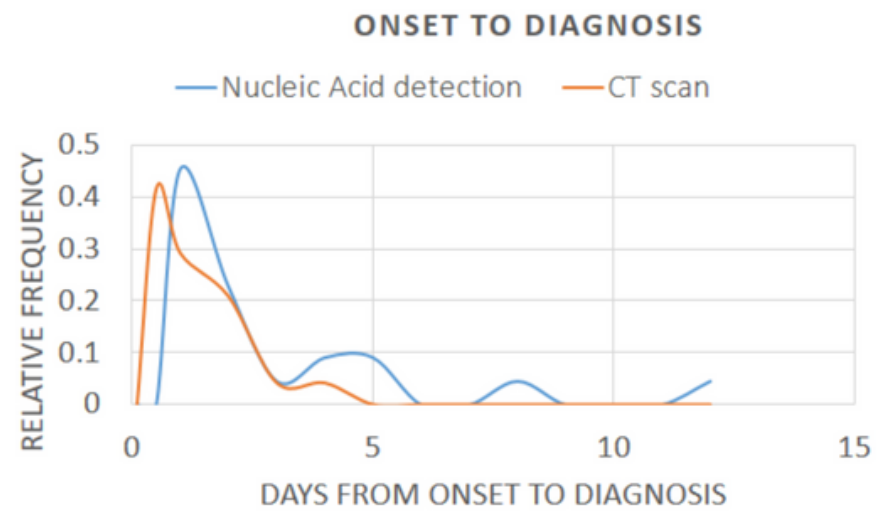

\section{Figure 3}

Time from the onset of symptoms to positive CT results and positive PCR results. 


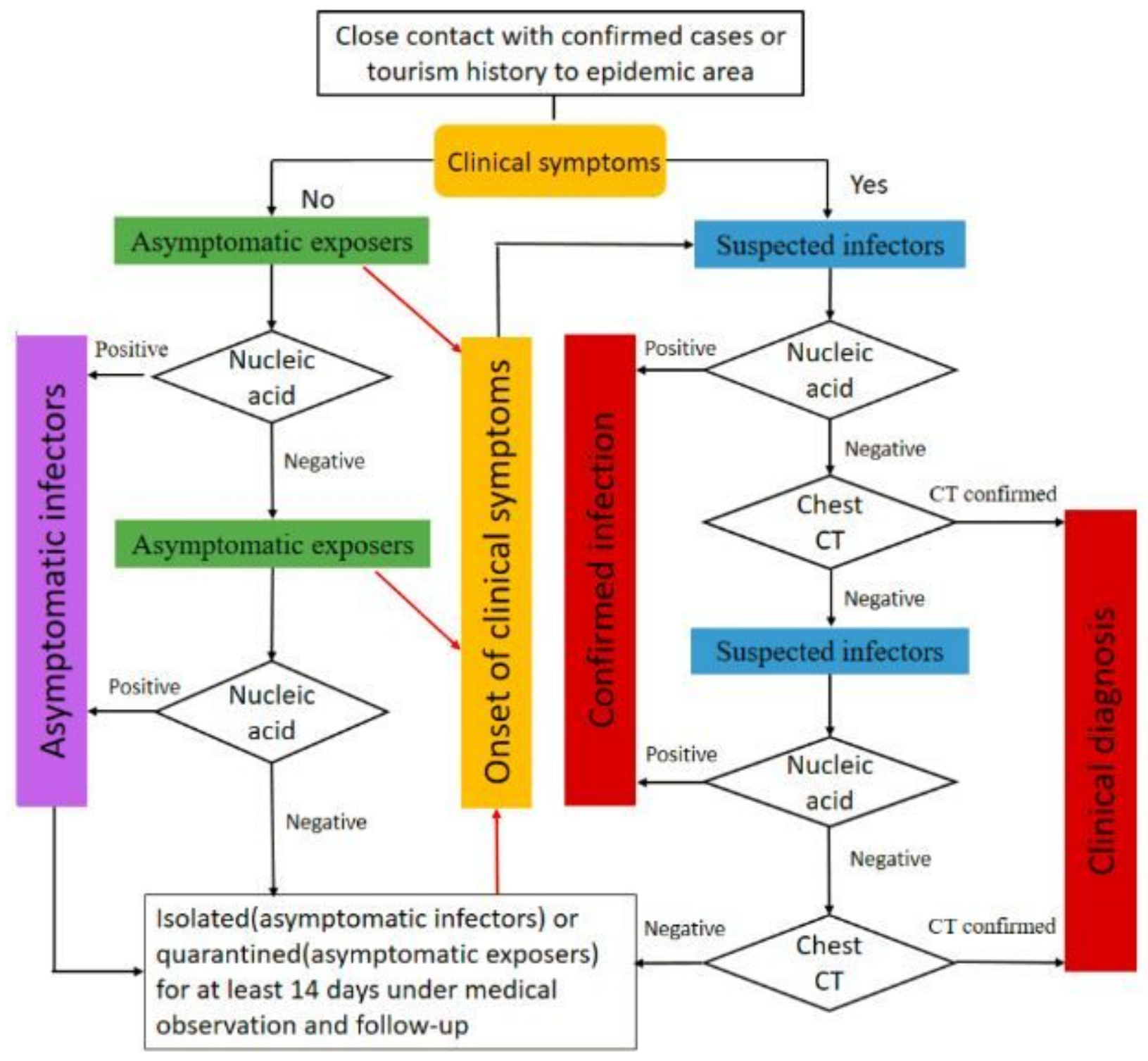

\section{Figure 4}

Disposal process after close contact with confirmed cases or tourism history of epidemic area, based on symptoms, nucleic acid test and chest CT examination.

\section{Supplementary Files}

This is a list of supplementary files associated with this preprint. Click to download.

- Epidemiologicalinvestigationofclusteroutbreaks2.25.docx 